\title{
Perioperative outcomes of zero ischemia radiofrequency ablation-assisted tumor enucleation for renal cell carcinoma: results of 182 patients
}

Chengwei Zhang ${ }^{1+}$, Xiaozhi Zhao ${ }^{1+}$, Suhan Guo ${ }^{2}$, Changwei $\mathrm{Ji}^{1}$, Wei Wang ${ }^{1}$ and Hongqian Guo ${ }^{1 *}$

\begin{abstract}
Background: To evaluate the perioperative outcomes of zero ischemia radiofrequency ablation-assisted tumor enucleation.

Methods: Patients undergoing zero ischemia radiofrequency ablation-assisted tumor enucleation were retrospectively identified from July 2008 to March 2013. The tumor was enucleated after RFA treatment. R.E.N.A.L., PADUA and centrality index (C-index) score systems were used to assess each tumor case. We analyzed the correlation of perioperative outcomes with these scores. Postoperative complications were graded with Clavien-Dindo system. Multivariate logistic regression analyses were used to assess risk of complications.

Results: Among 182 patients assessed, median tumor size, estimated blood loss, hospital stay and operative time were $3.2 \mathrm{~cm}$ (IQR 2.8-3.4), $80 \mathrm{ml}$ (IQR 50-120), 7 days (IQR 6-8) and $100 \mathrm{~min}$ (IQR 90-120), respectively. All three scoring systems were strongly correlated with estimated blood loss, hospital stay and operative time. We found 3 (1.6\%) intraoperative and 23 (12.6\%, 13 [7.1\%] Grade 1 and 10 [5.5\%] Grade 2 \& 3a) postoperative complications. The median follow-up was 55.5 months (IQR 45-70). Additionally, the complexities of R.E.N.A.L., PADUA and C-index scores were significantly correlated with complication grades $(P<0.001 ; P<0.001 ; P<0.001$; respectively). As the representative, R.E. N.A.L. score was an independent predictive factor for postoperative complications and patients with a high complexity had an over 24-fold higher risk compared to those with a low complexity (OR 24.360, 95\% Cl 4.412-134.493, $P<0.001)$.

Conclusions: Zero ischemia radiofrequency ablation-assisted tumor enucleation is considered an effective nephron-sparing treatment. Scoring systems could be useful for predicting perioperative outcomes of radiofrequency ablation-assisted tumor enucleation.
\end{abstract}

Keywords: Zero ischemia, Radiofrequency ablation, Tumor enucleation, Renal cell carcinoma, Nephrometry scoring systems

\section{Background}

Increasing numbers of small and incidental renal tumors have been detected with the enhancement of imaging technology. The estimated incidence of renal cancers is $5 \%$ among all tumors for males and $3 \%$ for females [1]. Nephron-sparing surgery (NSS) has been the recommended

\footnotetext{
* Correspondence: dr.ghq@nju.edu.cn

${ }^{\dagger}$ Equal contributors

${ }^{1}$ Department of Urology, Nanjing Drum Tower Hospital, Medical School of Nanjing University, 321 Zhongshan Rd., Nanjing 210008, People's Republic of China

Full list of author information is available at the end of the article
}

method to treat cT1a and T1b renal tumors to preserve renal function $[2,3]$. However, traditional NSS is considered to have some concerns, including hemostasis, tumor margin status, the collecting system invasion, renal vasculature clamping and hypothermia deployment.

Excision of the tumor with a substantial margin of normal parenchyma is the standard technique in partial nephrectomy and may reduce the risk of local recurrence [4]. To preserve more kidney parenchyma and avoid major bleeding, simple tumor enucleation (TE) was introduced in 2006. TE is a safe and acceptable

(c) The Author(s). 2018 Open Access This article is distributed under the terms of the Creative Commons Attribution 4.0 International License (http://creativecommons.org/licenses/by/4.0/), which permits unrestricted use, distribution, and 
treatment for NSS [5]. Moreover, the oncologic result with TE is similar to that with radical nephrectomy for treatment of both T1a and T1b renal cell carcinoma (RCC) [6].

As a result of hilar clamping, renal function will be influenced to a certain extent after ischemia with traditional NSS or simple TE, which is more important to the patients who suffer from solitary kidney. Radiofrequency ablation (RFA) has been used in medical field for more than 75 years [7]. The combination of RFA and NSS began in 2003, with no need for clamping the renal pedicle [8]. We reported our technique of RFA-assisted TE for renal tumors in 2012. Hemorrhage can be controlled to some extent and ischemia can be avoided to better protect renal function [9]. Therefore, we can achieve zero ischemia within our TE process.

Nephrometry scoring systems were recently created to predict surgical outcomes after partial nephrectomy. The R.E.N.A.L. and PADUA nephrometry scoring systems contain analogous elements, including tumor size, tumor depth, proximity or aggressiveness to the collecting system, tumor position (anterior or posterior plane) and tumor location in terms of polarity or relation to renal hilum $[10,11]$. Differently, The centrality index (CIndex) indicates tumor size and proximity relative to the renal hilum, which provides a measurement of tumor centrality [12]. Both R.E.N.A.L and C-index were found associated with decreased estimated glomerular filtration rate (eGFR) after partial nephrectomy [13, 14]. Satasivam et al.'s report figured out R.E.N.A.L score would predict histological features of tumor aggressiveness [15]. As a vital variable of standard NSS surgery, ischemia time was proved to have strong relationship with all three nephrometry score systems $[16,17]$. In spite TE has been widely approved for treatment of RCC, few studies focus on nephrometry scores to evaluate clinical outcomes after TE.

Therefore, we attempt to evaluate the perioperative outcomes of RFA-assisted TE for RCC in our single institute and associate the use of nephrometry scoring systems for predicting the perioperative complications.

\section{Methods}

\section{Patients}

We retrospectively identified consecutive patients who underwent RFA-assisted TE via an open or laparoscopic approach for a single renal tumor in our institution between July 2008 and March 2013. Patients with pathologically confirmed RCC were included. In addition to RFA assisted TE, simple TE and simple RFA are both our choices in the treatment for RCC. In this study all the selected patients were undergoing RFA assisted TE. All patients were informed of the option and all provided signed informed consent to be in the study, which was approved by the local ethics committee.

\section{Measurements}

Preoperatively, all tumors seen on enhanced computerized tomography $(\mathrm{CT})$ or magnetic resonance imaging (MRI) were scored by three senior urologists with different degrees of expertise in terms of the scoring systems. Final scores had interobserver concordance. Tumor stage was determined by the 2010 tumor-node-metastasis classification [4]. Ultrasonography and CT or MRI of the abdomen were performed preoperatively, as was chest X-ray, testing for serum creatinine level and other examinations. The eGFR was calculated by the modified Modification of Diet in Renal Disease equation (MDRD) before and after surgery [18]. Estimated blood loss, operative time and hospital stay were recorded.

Complexity levels of each nephrometry scoring system were defined as follows: R.E.N.A.L Scores ranged from 4 to 12 points. A score of 4,5 or 6 indicated a lesion of low complexity, and 10,11 or 12 indicated the highest complexity [10]. PADUA scores ranged from 6 to 14 . Tumor with a score of 6 or 7 was considered as low complexity while a score above 9 was high complexity [11]. For the C-index system, tumors were separated into 2 categories of greater than 2.5 (low complexity) or less than 2.5 (high complexity) [13].

\section{Zero ischemia RFA-assisted TE}

Our zero ischemia RFA-assisted TE technique was previously described [9]. All patients were under general anesthesia. The laparoscopic or open approach was via a retroperitoneal or transperitoneal route. The kidney was completely separated from perirenal fat and the renal pedicle was isolated. We localized the tumor by direct vision or intraoperative open or laparoscopic ultrasonography. Before RFA the tumor was biopsied percutaneously (17-gauge TruCore).

The electrode was inserted into the tumor via a percutaneous or laparoscopic approach, under the guidance of intraoperative ultrasonography. RFA was performed by the Cool-tip system, which was controlled by a feedback algorithm. One to three cycles were used, depending on tumor size and depth.

TE was performed with an open or laparoscopic approach with the renal hilum not clamped. Toward the pseudocapsule (PS), we incised the kidney capsule next to the lesion. The surgical plane was determined by the surgeon's choice. Blunt dissection was used to enucleate the tumors. The rim of the normal renal parenchyma was not visible. Bleeding control involved bipolar coagulation with a $1-\mathrm{cm}$ electrode for several minutes. The parenchymal defect remained open but covered with fibrin glue; the opening of the calyces was ligated by 
running or single suture with 3-zero monofilament. A single surgeon (HG) performed all surgeries.

\section{Follow-up}

The follow-up protocol at our institution comprised a clinical visit and physical examination, as well as contrast enhanced $\mathrm{CT}$ at 7 days, 3, 6 months and then every 6 months thereafter sequentially. Patients with renal insufficiency or contrast agent allergy were followed by enhanced MRI.

\section{Statistical analysis}

Data are presented as mean (SD), median (interquartile range [IQR]) or number (\%). All demographic data, including continuous and variables, was analyzed by independent chi-square test. Multivariate logistic regression analysis was used to determine variables predicting perioperative incidences of complications. Odds ratios (ORs) and $95 \%$ confidence intervals (95\% CIs) were calculated. Spearman's nonparametric method was used for correlation analysis because of nonnormal distribution of scores. All statistical analyses involved use of SPSS 18.0 (SPSS Inc., Chicago, IL). $P<0.05$ was considered statistically significant.

\section{Results}

Patients' demographics are demonstrated in Table 1. We identified 182 patients with perioperative imaging data (125 men [68.7\%]; mean [SD] age 57.6 [SD 11.0]; mean body mass index $23.3 \mathrm{~kg} / \mathrm{m}^{2}$ [SD 4.2]); the approach was laparoscopic for 170 patients (93.4\%) and open for 12 (6. 6\%); 115 (63.2\%) patients suffered from American Society of Anesthesiologists (ASA) scores of I or II and 67 (36.8\%) was scores of III. The median (IQR) tumor size was $3.2 \mathrm{~cm}(2.8-3.9)$ and most $(73.1 \%, n=133)$ were more than $50 \%$ exophytic. The median (IQR) operative time was $100 \mathrm{~min}$ (90-120 $\mathrm{min})$, median estimated blood loss $80 \mathrm{ml}(50-120 \mathrm{ml})$ and median hospital stay 7 days (6-8 days). No residual tumor was found on enhanced CT or MRI after surgery. Additionally, for most patients $(n=133,73.1 \%)$, tumors were clear-cell RCC on histopathology. No viable tumor cells were identified on the parenchymal side and the PS was undamaged in all cases. The median follow-up was 55 . 5 months (IQR 45-70). Totally there were 11 deaths occurring during the follow-up period, in which 2 were related to renal cancer. Distant metastasis developed in five patients at 18 and 35 months after surgery and they died at 38 and 55 months after surgery, respectively. Three patients suffered from lung metastasis and the other two were bone metastasis.

After calculating tumor scores on preoperative imaging, we associated the R.E.N.A.L., PADUA and C-Index scores with some clinical variables (Table 2). For all three scoring
Table 1 Characteristics of patients undergoing radiofrequency ablation (RFA)-assisted tumor enucleation (TE) for renal cell carcinoma $(n=182)$

\begin{tabular}{|c|c|}
\hline Age, years, mean (SD) & $57.6(11.0)$ \\
\hline Male gender & $125(68.7)$ \\
\hline Body mass index, $\mathrm{kg} / \mathrm{m}^{2}$, mean (SD) & $23.3(4.2)$ \\
\hline Operative time, min, median (IQR) & $100(90-120)$ \\
\hline Estimated blood loss, ml, median (IQR) & $80(50-120)$ \\
\hline Hospital stay, days, median (IQR) & $7(6-8)$ \\
\hline Tumor size, cm, median (IQR) & $3.2(2.8-3.9)$ \\
\hline Right tumor laterality & $102(55.7)$ \\
\hline Follow-up, months, median (IQR) & $55.5(45-70)$ \\
\hline \multicolumn{2}{|l|}{ ASA score } \\
\hline$<\|$ & $115(63.2)$ \\
\hline$>I I I$ & $67(36.8)$ \\
\hline \multicolumn{2}{|l|}{ eGFR, ml/min/1.73m², mean (SD) } \\
\hline Pre-operation & $64.3(18.1)$ \\
\hline 12 months post-operation & $60.8(17.3)$ \\
\hline \multicolumn{2}{|l|}{ Tumor location } \\
\hline$>50 \%$ exophytic & $133(73.1)$ \\
\hline$<50 \%$ exophytic & $41(22.5)$ \\
\hline Entirely endophytic & $8(4.4)$ \\
\hline \multicolumn{2}{|l|}{ Tumor position } \\
\hline Anterior & $85(46.7)$ \\
\hline Posterior & $97(53.3)$ \\
\hline \multicolumn{2}{|l|}{ Surgical approach } \\
\hline Laparoscopic & $170(93.4)$ \\
\hline Open & $12(6.6)$ \\
\hline \multicolumn{2}{|l|}{ Histology, no. patients } \\
\hline Clear cell renal cell carcinoma & 133 \\
\hline Papillary & 13 \\
\hline Oncocytoma & 10 \\
\hline Angiomyolipoma & 15 \\
\hline Chromophobe renal cell cancer & 8 \\
\hline Unclassified renal cell cancer & 3 \\
\hline
\end{tabular}

Data are no. (\%) unless indicated. IQR Interquartile range, ASA American Society of Anesthesiologists, eGFR Estimated glomerular filtration rate

systems, in which R.E.N.A.L. score complexity played the most significant role $(P<0.001)$, estimated blood loss, operative time and hospital stay but not eGFR change differed by score complexity. All scores and their complexities were strongly correlated with estimated blood loss, operative time and hospital stay (Table 3). However, correlation coefficients with eGFR change in absolute value or percentage were less than 0.2 , suggesting a weak correlation.

We evaluated perioperative complications, found 3 intraoperative complications and 23 postoperative ones. 
Table 2 Association between nephrometry scores and clinical outcome variables

\begin{tabular}{|c|c|c|c|c|c|}
\hline Nephrometry scores & No. (\%) & $\begin{array}{l}\text { Estimated blood loss, } \\
\mathrm{ml} \text {, median (IQR) }\end{array}$ & $\begin{array}{l}\text { Operative time, min, } \\
\text { median (IQR) }\end{array}$ & $\begin{array}{l}\text { Hospital stay, days, } \\
\text { median (IQR) }\end{array}$ & $\begin{array}{l}\text { Change in eGFR, } \\
\text { median (IQR) }\end{array}$ \\
\hline \multicolumn{6}{|l|}{ R.E.N.A.L. score } \\
\hline Low (4-6) & 132 & $70(50,100)$ & $100(90,110)$ & $6(5.25,8)$ & $-4.05(-6.88,-1.3)$ \\
\hline Moderate (7-9) & 43 & $120(75,160)$ & $120(100,130)$ & $8(6,9)$ & $-3.7(-5.3,-0.9)$ \\
\hline High (10-12) & 7 & $210(170,250)$ & $150(120,160)$ & $10(8,10)$ & $-0.9(-3.3,1.3)$ \\
\hline$P$ value & & $<0.001$ & $<0.001$ & $<0.001$ & 0.136 \\
\hline \multicolumn{6}{|l|}{ PADUA score } \\
\hline Low (6-7) & 108 & $70(50,90)$ & $100(90,110)$ & $6(5.25,8)$ & $-4.2(-6.72,-1.3)$ \\
\hline Moderate (8-9) & 64 & $100(61.25,148.75)$ & $102.5(90,127.5)$ & $8(6,9)$ & $-3.4(-6.05,-1.12)$ \\
\hline High (10-13) & 10 & 190 (127.5220) & $130(103.75,156.25)$ & $8.5(6.5,10)$ & $-1.25(-3.35,1.95)$ \\
\hline$P$ value & & $<0.001$ & 0.002 & 0.005 & 0.112 \\
\hline \multicolumn{6}{|l|}{ C-index score } \\
\hline Low (> 2.5) & 116 & $70(50,100)$ & $100(90,113.75)$ & $6(5,8)$ & $-3.8(-6.72,-0.95)$ \\
\hline High $(<2.5)$ & 66 & $102.5(75,162.5)$ & $102.5(95,130)$ & $7.5(6,9)$ & $-3.75(-5.98,-0.9)$ \\
\hline$P$ value & & $<0.001$ & 0.005 & 0.006 & 0.921 \\
\hline
\end{tabular}

Boldface means the data is significant $(P<0.05)$

All the postoperative complications were classified by the Clavien-Dindo grading system, which included 17 (74\%) Grade 1-2 complications (Grade 1: 18, Grade 2: 1) and 6 (26\%) Grade 3a complications (Table 4). The major complications (Grade 3a) contained urinary leakage and perinephric urinoma. At the same time, we identified strong correlation of complexities between postoperative complications and all the three systems $(P<0.001$, Table 5$)$, which meant high systems score was significantly associated with high incidence of complications. R.E.N.A.L. score had a most significant correlation coefficient $(\rho=0.376)$. To evaluate potential preoperative risk factors associated with postoperative complications, multivariate logistic regression analysis was performed. As the representative of score systems, R.E.N.A.L. score was the only independent predictive factor of the occurrence of postoperative complications (Table 6). Patients with a high complexity (R.E.N.A.L. score 10-12) had an over 24fold higher risk compared with those with a low complexity (R.E.N.A.L. score 4-6).

\section{Discussion}

We succeeded to perform zero ischemia RFA-assisted TE in 182 patients. We evaluated the follow-up of our
RFA-assisted TE and the association of these scoring systems with perioperative outcomes. All three scoring systems were strongly correlated with estimated blood loss, hospital stay and operative time. The complexity of the scoring systems was significantly associated with postoperative complication grades. Additionally, R.E.N. A.L. scores were an independent predictive factor for postoperative complications and patients with a high complexity had an over 24-fold higher risk compared to those with a low complexity (OR 24.360, 95\% CI 4.412134.493, $P<0.001)$. Zero ischemia RFA-assisted TE is considered a safe and effective nephron-sparing treatment. Scoring systems could be useful for predicting perioperative outcomes of RFA-assisted TE.

Simple TE has been found a safe and acceptable NSS treatment. Carini et al. evaluated the safety and efficacy of simple $\mathrm{TE}$ as a conservative treatment in the early twentieth century: among 232 patients undergoing TE for sporadic, unilateral, pathologically confirmed pT1a RCC, no major complications were found [5]. Likewise, TE was associated with the same progressionfree survival and cancer-specific survival as with racial nephrectomy $(\mathrm{RN})$ for both $\mathrm{T} 1 \mathrm{a}$ and $\mathrm{T} 1 \mathrm{~b} \mathrm{RCC}$ [6]. In terms of adverse events, the rate was $16 \%$,

Table 3 Correlation between nephrometry scores and clinical outcomes

\begin{tabular}{|c|c|c|c|c|c|}
\hline Tumor characteristics and scores & Estimated blood loss & Operative time & Hospital stay & Absolute change in eGFR & Percentage change in eGFR \\
\hline R.E.N.A.L. score & $0.438^{* *}$ & $0.252^{* *}$ & $0.210^{*}$ & 0.072 & 0.077 \\
\hline PADUA score & $0.373^{* *}$ & $0.264^{* *}$ & $0.241^{* *}$ & 0.087 & 0.105 \\
\hline C-Index Score & $0.407^{* *}$ & $0.311^{* *}$ & $0.203^{*}$ & 0.007 & 0.035 \\
\hline
\end{tabular}

Data are Spearman correlation coefficients, $\rho$

${ }^{*} P<0.05$

${ }^{*} P<0.001$ 
Table 4 Perioperative complications characteristics

\begin{tabular}{ll}
\hline Complications & No. patients \\
\hline Intraoperative complications & 3 \\
Blood loss requiring transfusion & 2 \\
Conversion & 1 \\
Postoperative complications & 23 \\
Clavien-Dindo Grade 1 & 13 \\
Pain & 10 \\
Hematuria & 2 \\
Renal infarction & 1 \\
Clavien-Dindo Grade 2 & 4 \\
Limited hematoma & 1 \\
Postoperative Fever & 3 \\
Clavien-Dindo Grade 3a & 6 \\
Urinary leakage (stent) & 5 \\
Perinephric urinoma (drainage) & 1 \\
\hline
\end{tabular}

with only $3 \%$ needing re-intervention [19]. TE and RN showed oncologic equivalence in a large cohort (about 1000 patients undergoing $\mathrm{RN}$ and $500 \mathrm{TE}$ ) in 16 centers [20].

The width of surgical margin seems the most striking difference between TE and traditional NSS. Continuous PS determines the oncologic safety of TE. In general, TE is performed by blunt dissection by using the natural cleavage plane between the tumor and the normal parenchyma. Among 90 consecutive patients undergoing TE, 67\% tumors were intact and uninvaded. Although the remaining patients showed signs of penetration within layers, only 6 showed penetration on the perirenal fat tissue side. The surgical margin was negative after TE in all cases [21].

Table 5 Correlation between nephrometry score complexity and postoperative complication grade

\begin{tabular}{|c|c|c|c|c|c|}
\hline \multirow{2}{*}{$\begin{array}{l}\text { Nephrometry } \\
\text { scores }\end{array}$} & \multicolumn{3}{|c|}{ Complication Grade } & \multirow[t]{2}{*}{$\rho^{a}$} & \multirow[t]{2}{*}{$P$ value } \\
\hline & Absent & Grade 1 & Grade 2 and $3 a$ & & \\
\hline \multicolumn{6}{|l|}{ R.E.N.A.L. score } \\
\hline Low (4-6) & 125 & 4 & 3 & 0.376 & $<0.001$ \\
\hline Moderate (7-9) & 31 & 7 & 5 & & \\
\hline High (10-12) & 3 & 2 & 2 & & \\
\hline \multicolumn{6}{|l|}{ PADUA score } \\
\hline Low (6-7) & 103 & 3 & 2 & 0.372 & $<0.001$ \\
\hline Moderate (8-9) & 53 & 7 & 4 & & \\
\hline High (10-13) & 3 & 3 & 4 & & \\
\hline \multicolumn{6}{|l|}{ C-Index score } \\
\hline Low (> 2.5) & 110 & 2 & 4 & 0.290 & $<0.001$ \\
\hline High $(<2.5)$ & 49 & 11 & 6 & & \\
\hline
\end{tabular}

apearman correlation coefficients

Boldface means the data is significant $(P<0.05)$
Table 6 Multivariate logistic regression predicting incidences of postoperative complications in patients undergoing RFAassisted TE

\begin{tabular}{|c|c|c|c|}
\hline Variable & OR & $95 \% \mathrm{Cl}$ & $P$ value \\
\hline Age & 0.997 & $0.954-1.042$ & 0.897 \\
\hline Gender & 1.875 & $0.512-6.868$ & 0.343 \\
\hline BMI & 1.955 & $0.672-6.118$ & 0.169 \\
\hline ASA score & 1.817 & $0.735-5.160$ & 0.278 \\
\hline Laterality & 2.499 & $0.657-9.501$ & 0.179 \\
\hline $\begin{array}{l}\text { Surgical approach } \\
\text { (laparoscopic vs open) }\end{array}$ & 1.744 & $0.832-2.011$ & 0.461 \\
\hline \multicolumn{4}{|l|}{ R.E.N.A.L Score } \\
\hline Low (4-6) & Reference & & \\
\hline Moderate (7-9) & 7.062 & $2.566-19.436$ & $<0.001$ \\
\hline High (10-12) & 24.360 & $4.412-134.493$ & $<0.001$ \\
\hline
\end{tabular}

RFA is considered a minimally invasive technique to treat renal tumor. In the past 10 years, percutaneous RFA was an effective treatment for patients, who survived with a small renal mass but had poor surgical condition [3]. Our institute started to treat renal tumors with RFA in 2005 [22]. We have identified that R.E.N.A. L. score is independently associated with occurrence of complications after RFA [23].

RFA-assisted TE has many advantages as compared with simple RFA or NSS. We have achieved zero ischemia during resection of renal tumors with RFA-assisted TE, which maximizes the prevention or delay of decreased renal function. Huang $J$ reported a randomized clinical trial in 2016 and compared the renal functional outcome between RFA assisted TE and conventional laparoscopic partial nephrectomy. Results showed zero ischemia RFA assisted TE presented better renal function preservation. Our results in this article proved that the functional outcomes were also associated with the nephrometry scores [24]. With RFA as a single procedure, our technique allows surgeons to remove the entire tumor, which can provide an accurate pathological result to identify positive or negative surgical margins. According to our previous report, we have proved the oncological safety for the technique of RFA assisted TE. No patient showed positive surgical margins. Microscopy revealed that the pseudocapsule was intact in all cases and no viable tumor cells were identified on the parenchymal side of the tumor [9].

Since our technique is performed without hilar clamping, we did not achieve good intraoperative bleeding control. However, we found little incidence of blood transfusion after surgery because of the superiority of RFA for hemostasis. In contrast, the incidence of urinary 
leakage seemed high as compared with conventional NSS or TE. We found 5 patients with prolonged urinary leakage, which might occur when the tumor was close to the calyces. We found that the nephrometry scoring systems could predict the incidence of complications. As the result, communication and counseling to patients with high nephrometry scores is important. Rosevear et al. similarly showed an association of the R.E.N.A.L. score and complications for patients undergoing partial nephrectomy [25]. Bruner et al. reported an association between R.E.N.A.L. score and urinary leakage after partial nephrectomy [26], which also could be concluded from our research. On the basis of Minervini's report about TE, PADUA score was associated with complications, especially Clavien-Dindo grade 3 surgical complications [27].

Our study has some limitations. Because of the short follow-up, we cannot evaluate the 5-years OS or CSS of RFA-assisted TE. Secondly, our study was retrospective. Randomized controlled studies about RFA-assisted TE should be performed to study this technique further.

\section{Conclusions}

Zero ischemia RFA-assisted TE is considered an oncologically safe technique to treat renal cancer, for both protect of renal function and low rate of perioperative complications. Nephrometry scoring systems represent a multifactorial approach to evaluate the renal masses and categorize patients undergoing RFA-assisted TE. From the strong relationship we found, scoring systems may give pertinent information about perioperative outcomes. Zero ischemia RFA-assisted TE is an effective option to treat renal carcinoma.

\section{Abbreviations}

95\% Cl: 95\% confidence interval; C-Index: Centrality index; CT: Computerized tomography; eGFR: Estimated glomerular filtration rate; HR: Hazard ratio; MRI: Magnetic resonance imaging; NSS: Nephron-sparing surgery; OR: Odds ratio; PS: Pseudocapsule; RCC: Renal cell carcinoma; RFA: Radiofrequency ablation; RN: Racial nephrectomy; TE: Tumor enucleation

\section{Availability of data and materials}

The datasets analyzed during the current study available from the corresponding author on reasonable request.

\section{Authors' contributions}

CZ: Protocol development, Acquisition of data, Statistical analysis, Manuscript writing. XZ: Protocol development, Acquisition of data, Statistical analysis, Manuscript editing. SG: Manuscript editing. CJ: Acquisition of data. WW: Acquisition of data. HG: Protocol development, Manuscript editing. All authors read and approved the final manuscript.

\section{Ethics approval and consent to participate}

In this study, we obtained the patient-informed consent of the involved patients before starting; the study was additionally approved by the Ethics Committee of Nanjing Drum Tower Hospital.

\section{Competing interests}

The authors declare that they have no competing interests.

\section{Publisher's Note}

Springer Nature remains neutral with regard to jurisdictional claims in published maps and institutional affiliations.

\section{Author details}

'Department of Urology, Nanjing Drum Tower Hospital, Medical School of Nanjing University, 321 Zhongshan Rd., Nanjing 210008, People's Republic of China. ${ }^{2}$ School of Public Health, Nanjing Medical University, Nanijng 210029, People's Republic of China.

Received: 22 September 2016 Accepted: 3 May 2018 Published online: 15 May 2018

\section{References}

1. Siegel R, Ma J, Zou Z, Jemal A. Cancer statistics, 2014. CA Cancer J Clin. 2014;64(1):9-29.

2. Uzzo RG, Novick AC. Nephron sparing surgery for renal tumors: indications, techniques and outcomes. J Urol. 2001;166(1):6-18.

3. Zagoria RJ, Pettus JA, Rogers M, Werle DM, Childs D, Leyendecker JR. Longterm outcomes after percutaneous radiofrequency ablation for renal cell carcinoma. Urology. 2011;77(6):1393-7.

4. Ljungberg B, Cowan NC, Hanbury DC, Hora M, Kuczyk MA, Merseburger AS, Patard JJ, Mulders PF, Sinescu IC, European Association of Urology Guideline G. EAU guidelines on renal cell carcinoma: the 2010 update. Eur Urol. 2010; 58(3):398-406

5. Carini M, Minervini A, Masieri L, Lapini A, Serni S. Simple enucleation for the treatment of PT1a renal cell carcinoma: our 20-year experience. Eur Urol. 2006:50(6):1263-8. discussion 1269-1271

6. Minervini A, Serni S, Tuccio A, Siena G, Vittori G, Masieri L, Giancane S, Lanciotti M, Khorrami S, Lapini A, et al. Simple enucleation versus radical nephrectomy in the treatment of pT1a and pT1b renal cell carcinoma. Ann Surg Oncol. 2012;19(2):694-700.

7. Triadafilopoulos $\mathrm{G}$. The great beyond: radiofrequency ablation for hemostasis. Endoscopy. 2014;46(11):925-6.

8. Jacomides L, Ogan K, Watumull L, Cadeddu JA. Laparoscopic application of radio frequency energy enables in situ renal tumor ablation and partial nephrectomy. J Urol. 2003;169(1):49-53. discussion 53

9. Zhao X, Zhang S, Liu G, Ji C, Wang W, Chang X, Chen J, Li X, Gan W, Zhang G, et al. Zero ischemia laparoscopic radio frequency ablation assisted enucleation of renal cell carcinoma: experience with 42 patients. J Urol. 2012;188(4):1095-101.

10. Kutikov A, Uzzo RG. The R.E.N.A.L. nephrometry score: a comprehensive standardized system for quantitating renal tumor size, location and depth. J Urol. 2009;182(3):844-53.

11. Ficarra V, Novara G, Secco S, Macchi V, Porzionato A, De Caro R, Artibani W. Preoperative aspects and dimensions used for an anatomical (PADUA) classification of renal tumours in patients who are candidates for nephronsparing surgery. Eur Urol. 2009;56(5):786-93.

12. Simmons MN, Ching CB, Samplaski MK, Park CH, Gill IS. Kidney tumor location measurement using the C index method. J Urol. 2010;183(5):1708-13.

13. Samplaski MK, Hernandez A, Gill IS, Simmons MN. C-index is associated with functional outcomes after laparoscopic partial nephrectomy. J Urol. 2010; 184(6):2259-63.

14. Kwon T, Jeong IG, Ryu J, Lee C, Lee C, You D, Kim CS. Renal function is associated with Nephrometry score after partial nephrectomy: a study using diethylene Triamine Penta-acetic acid (DTPA) renal scanning. Ann Surg Oncol. 2015;22(Suppl 3):1594-600.

15. Satasivam P, Sengupta S, Rajarubendra N, Chia PH, Munshey A, Bolton D. Renal lesions with low R.E.N.A.L nephrometry score are associated with more indolent renal cell carcinomas (RCCS) or benign histology: findings in an Australian cohort. BJU Int. 2012;109(Suppl 3):44-7.

16. Spaliviero M, Poon BY, Aras O, Di Paolo PL, Guglielmetti GB, Coleman CZ, Karlo CA, Bernstein ML, Sjoberg DD, Russo P, et al. Interobserver variability of R.E.N.A.L., PADUA, and centrality index nephrometry score systems. World J Urol. 2015;33(6):853-8.

17. Bylund JR, Gayheart D, Fleming T, Venkatesh R, Preston DM, Strup SE, Crispen PL. Association of tumor size, location, R.E.N.A.L., PADUA and centrality index score with perioperative outcomes and postoperative renal function. J Urol. 2012;188(5):1684-9.

18. Levey AS, Bosch JP, Lewis JB, Greene T, Rogers N, Roth D. A more accurate method to estimate glomerular filtration rate from serum creatinine: a new 
prediction equation. Modification of diet in renal disease study group. Ann Intern Med. 1999;130(6):461-70.

19. Minervini A, Vittori G, Lapini A, Tuccio A, Siena G, Serni S, Carini M. Morbidity of tumour enucleation for renal cell carcinoma (RCC): results of a single-Centre prospective study. BJU Int. 2012;109(3):372-7. discussion 378

20. Minervini A, Ficarra V, Rocco F, Antonelli A, Bertini R, Carmignani G, Cosciani Cunico S, Fontana D, Longo N, Martorana G, et al. Simple enucleation is equivalent to traditional partial nephrectomy for renal cell carcinoma: results of a nonrandomized, retrospective, comparative study. J Urol. 2011;185(5):1604-10.

21. Minervini A, di Cristofano C, Lapini A, Marchi M, Lanzi F, Giubilei G, Tosi N, Tuccio A, Mancini M, della Rocca C, et al. Histopathologic analysis of peritumoral pseudocapsule and surgical margin status after tumor enucleation for renal cell carcinoma. Eur Urol. 2009;55(6):1410-8.

22. Ji C, Li X, Zhang S, Gan W, Zhang G, Zeng L, Yan X, Liu T, Lian H, Guo H. Laparoscopic radiofrequency ablation of renal tumors: 32 -month mean follow-up results of 106 patients. Urology. 2011;77(4):798-802.

23. Chang X, Ji C, Zhao X, Zhang F, Lian H, Zhang S, Liu G, Zhang G, Gan W, Li X, et al. The application of R.E.N.A.L. nephrometry scoring system in predicting the complications after laparoscopic renal radiofrequency ablation. J Endourol. 2014;28(4):424-9.

24. Huang J, Zhang J, Wang Y, Kong W, Xue W, Liu D, Chen Y, Huang Y. Comparing zero ischemia laparoscopic radio frequency ablation assisted tumor enucleation and laparoscopic partial nephrectomy for clinical T1a renal tumor: a randomized clinical trial. J Urol. 2016;195(6):1677-83.

25. Rosevear HM, Gellhaus PT, Lightfoot AJ, Kresowik TP, Joudi FN, Tracy CR Utility of the RENAL nephrometry scoring system in the real world: predicting surgeon operative preference and complication risk. BJU Int. 2012;109(5):700-5.

26. Bruner B, Breau RH, Lohse CM, Leibovich BC, Blute ML. Renal nephrometry score is associated with urine leak after partial nephrectomy. BJU Int. 2011; 108(1):67-72.

27. Minervini A, Vittori G, Salvi M, Sebastianelli A, Tuccio A, Siena G, Masieri L, Gacci M, Lapini A, Serni S, et al. Analysis of surgical complications of renal tumor enucleation with standardized instruments and external validation of PADUA classification. Ann Surg Oncol. 2013;20(5):1729-36.

Ready to submit your research? Choose BMC and benefit from:

- fast, convenient online submission

- thorough peer review by experienced researchers in your field

- rapid publication on acceptance

- support for research data, including large and complex data types

- gold Open Access which fosters wider collaboration and increased citations

- maximum visibility for your research: over $100 \mathrm{M}$ website views per year

At BMC, research is always in progress.

Learn more biomedcentral.com/submissions 